\title{
Peer Victimization among High School Children: A Prevalence Study
}

\author{
Drakshayini T. Tippanagoudra ${ }^{1 *}$, Manjula Patil ${ }^{2}$ and Saraswati Hunshal ${ }^{2}$ \\ ${ }^{1}$ Department of Human Development and Family Studies, College of Community Science, \\ UAS, Dharwad, Karnataka, India \\ ${ }^{2}$ Junior Scientist AICRP (CD), Research Complex UAS, Dharwad, Karnataka, India \\ *Corresponding author
}

\section{A B S T R A C T}

\begin{tabular}{|l|}
\hline Ke y w o r d s \\
Peer victimization, \\
Prevalence, \\
Bullying, \\
Aggressive \\
Behaviour \\
\hline Article Info \\
\hline $\begin{array}{l}\text { Accepted: } \\
\text { 25 February } 2020 \\
\text { Available Online: } \\
\text { 10 March } 2020\end{array}$ \\
\hline
\end{tabular}

Peer victimization is major social problem affecting children and adolescents in all parts of the world. A study was conducted in University of Agricultural Sciences Dharwad to know the prevalence and levels of peer victimization among high school children selected from Dharwad taluk, Dharwad district of Karnataka. Self structured questionnaire and multidimensional peer victimization scales were used to elicit required information from children. Percentages and t-test were used to analyze the data. The results related to prevalence showed that, out of total sample (1150) 265 children were found to be victimized. Among them, about 53.21 per cent of children were from rural area and 46.79 per cent of children were from urban area. In rural area, about 28.30 per cent of children were studying in government schools and 24.91 per cent of children studying in private schools were experienced victimization. In case of urban area, victimization was found to be more $(25.28 \%$ ) among children studying in government schools followed by children studying in private schools $(21.51 \%)$. Among them most of boys studying in $8^{\text {th }}$ class were found to be more victimized followed by $9^{\text {th }}$ and $10^{\text {th }}$ class children in both rural and urban area. Majority of rural $(68.08 \%)$ and urban $(58.87 \%)$ children were found in moderate level of victimization followed by low level and high level of victimization. So, there is need for school based educational programs and public awareness campaigns, including print, electronic and social media with emphasis to rural and urban area and strict enforcement of policies to make the school environment safe is necessary.

\section{Introduction}

Young children begin their lives in social world of their families however, as they grow older they are introduced to social world of peers and spend their time with children of their own age. When children move out from family to child-care centers, school and community, they begin to form attachments and friendships through their play. Peer group sharply increases during the transition from childhood to adolescents. Peers of similar age, background and social status provides different opportunities to discuss feelings and expand knowledge, language and social roles. Peers can promote good academic performance, encouragement to involve in extracurricular activities. 
As a child gets older he/she needs approval of peers more than their parents, strong peer relation helps teens to achieve two of the more critical tasks: finding independence from parents and develop their own personal identity. Prior to adolescence, a child is controlled by parents, but in adolescence, where teens begin to select their own friends, spend more time with them and often do without parental supervision they begin to share bonds with their friends. During the time of worry or upset peers become first line of support. Because acceptance by a peer group becomes so important, teens modify their speech, dress, behavior, choices and activities in order to become more similar to their peers, this provides them a sense of security and acceptance into their chosen group. But sometimes rejection from peers may also have negative effect on child it leads to victimization/bullying.

Peer victimization is commonly labeled as harassment/bullying. School bullying and peer victimization are major social problems affecting children and adolescents in all parts of the world. Peer victimization or bullying is a repeated aggressive or threatening behavior between peers of unequal size or power, not including teasing in a friendly or playful way (Dake et al., 2003). Peer victimization is the experience among children of being a target of the aggressive behavior of other children, who are not siblings and not necessarily age mates. Bullying is typically defined as aggressive peer to peer behavior in which there is an intention to harm or disturb the victim, the aggression occurs repeatedly over a time and there is an imbalance of power, with a powerful person or group attacking a less powerful one (Olweus, 1993).

Globally, slightly more than one in three students aged 13-15 years have experienced bullying (UNICEF, 2018). In India, on an average 60 per cent (boys 63.9\%, girls 53\%) of children aged 8-14 years of children have been bullied (Ramya and Kulkarni, 2011).

Report on bullying by gender shows that, 61 per cent of males and 34 per cent of females have experienced victimization (Anonymous, 2019). Based on this information the study was carried out to know the prevalence and levels of peer victimization among rural and urban high school children.

\section{Materials and Methods}

\section{Population and sample}

The population for the study comprised of rural and urban high school students studying in government and private schools of Dharwad taluk.

List of high schools was obtained from Block Education Officer of Dharwad taluk. There were 233 high schools (Government-183, aided-20 and unaided-30) in urban locality and 208 high schools (Government-70, aided-54 and unaided-84) in rural locality.

Six schools, i.e. 3 per cent of school from rural and urban areas were randomly selected for the study purpose. Permission was obtained from Block Education Officer to visit schools and Principals of selected schools were contacted to obtain the permission to carry out research work.

Sample for the study was selected in two stages. In the first stage to study the prevalence, total class students studying in VIII, IX and X grades of two government and two private Kannada medium schools were selected from rural and urban areas of Dharwad taluk. Totally 1150 children (Rural582 Urban-568) were screened for prevalence of peer victimization. In the second stage, only children who reported to be "victimized" they were selected for further study. 


\section{Tools used}

Self structured questionnaire was used to know about being bullied/victimized in the past couple of month. The questions which are included in the questionnaire are

"Have you been hurt like being hit/ kicked by another student?"

Has anyone teased you/called you name?"

Has anyone damage your peer relationship?"

Has anyone took something without your permission?".

The responses were coded as "yes" or "no". Children who answered 'yes' even for one question were considered as peer victimized children. To know the levels of peer victimization the multidimensional peer victimization scale developed by Mynard and Joseph (2000) was used. The scale consisted of 20 items indicating 4 types of victimization like physical (5 items), verbal (5 items), social (5 items) and attack on property (5 items). For each item 3 alternatives are given like 0 - not at all, 1- once and 2- more than once. Based on obtained total score an individual child was classified into three groups as low (0-18), moderate (19-26) and high (27-40).

\section{Procedure}

The study was conducted in two phases

\section{Phase 1}

During first phase respective class teachers were contacted and explained about the purpose of the study. The concept of peer victimization was explained among 1150 children to make them understand about what is peer victimization? and later they were asked to answer the questions related to peer victimization. Children who answered 'yes' even for one question were considered as peer victimized children and later the general information schedule was distributed to collect the data on personal and familial information.

\section{Phase 2}

During second phase based on the information given by students the victimization was observed that, in rural area 141 children were found to be victimized out of 582 children and in urban area 124 children were found to be victimized out of 568 children. To avoid disturbance in the regular classes separate arrangement was made in the school premises and then the victimized children from each class were gathered at one place during off periods and were explained about the purpose of the study as to seek co-operation and provide correct information. Socio economic status scale was administered to victimized children to know their SES level and to know the type of victimization multidimensional peer victimization scale was administered.

\section{Results and Discussion}

The data in the table 1 shows phase 1 distribution of rural and urban children according to child characteristics such as class, gender and type of school. With regard to class, in rural area, 34.20 per cent of children were studying in $8^{\text {th }}$ class, 33.33 per cent of children were studying in $9^{\text {th }}$ class and 32.47 per cent of children were studying in $10^{\text {th }}$ class. In case of urban area, 34.15 per cent of children studying in $8^{\text {th }}$ class followed by 33.45 per cent of children studying in $9^{\text {th }}$ class and 32.40 per cent of children were studying in $10^{\text {th }}$ class. The results in relation to gender showed that, in rural locality, 50.86 per cent of children were boys and 49.14 per cent of children were girls. In case of urban locality, 50.18 per cent of children were boys and 49.82 per cent of children were girls. The 
results on type of school revealed that, in rural locality, most $(52.23 \%)$ of children belonged to private schools followed by 47.77 per cent of children were studying in government schools. In case of urban locality, 49.30per cent of children were belonged to private schools and 50.70 per cent of children were studying in government schools.

Prevalence of peer victimization among rural and urban high school children given in table 2 reveals that, in rural area, out of 582 children, 53.21 per cent of children were found to be victimized among these, 28.30 per cent of children were studying in government schools and 24.91 per cent of children were studying in private schools. About 10.18 per cent of children studying in $8^{\text {th }}$ class of government schools were found to be victimized among them, most $(5.66 \%)$ of them were boys and 4.52 per cent of girls were found to be victimized. With regard to $9^{\text {th }}$ class children, 9.82 per cent were found to be victimized among them, most $(5.66 \%)$ of the victimization was found in boys than in girls $(4.16 \%)$. With respect to $10^{\text {th }}$ class students, 8.30 per cent of children were victimized among which, 4.53 per cent were boys and 3.77 per cent were girls. In private schools, 9.05 per cent of victimization was found in $8^{\text {th }}$ class children among which, most $(5.28 \%)$ of them were boys compared to girls $(3.77 \%)$. Among $9^{\text {th }}$ class children, 8.68 per cent of victimization was found of which, 4.91 per cent were boys and 3.77 per cent were girls. About 7.18 per cent of victimization was found in children studying in $10^{\text {th }}$ class among which, 4.16 per cent were boys and 3.02 per cent were girl students.

In case of urban area, out of 568 high school children, 46.79 per cent of children were found to be victimized among these, 25.28 per cent belonged to government schools and 21.51 per cent of children were studying in private schools. In government schools children, most $(9.05 \%)$ of children studying in $8^{\text {th }}$ class and $9^{\text {th }}$ class were found to be equally victimized among which, 5.28 per cent of boys and 3.77 per cent of girl students experienced victimization. About 7.18 per cent of victimization was found in $10^{\text {th }}$ class children, where 4.16 per cent was boys and 3.02 per cent was girl students. In private schools, most $(7.92 \%)$ of children studying in $8^{\text {th }}$ class had experienced victimization in that, 4.52 per cent of boys and 3.40 per cent of girls experienced victimization. Among $9^{\text {th }}$ class children, 7.18 per cent of victimization was found among which, 4.16 per cent of boys and 3.02 per cent of girls were found to be victimized. About 6.41 per cent of victimization was experienced by $10^{\text {th }}$ class students among which, 3.77 per cent of boys and 2.64 per cent of girls were found to be victimized.

Rural children were more adversely affected by peer victimization and severity of victimization was higher among boys of government schools than private schools boys this may be due to negligence in case of appropriate disciplinary practices by teachers of government schools in rural areas and also by parents of children studying in government schools. The results are in line with study conducted by Finkelhore et al., (2007) which revealed that children from rural area were more victimized $(53.21 \%)$ than children from urban. Another study conducted by Delfabbro et al., (2006) reported significant interaction between government and private school boys.

Results related to distribution of phase 2 victimized children according to child characteristics such as class, gender, ordinal position, academic achievement and type of school are presented in table 3a. With regard to class of the children, in rural area, 36.17 per cent of victimized children were studying in $8^{\text {th }}$ class, 34.75 per cent of children were studying in $9^{\text {th }}$ class and 29.08 per cent of 
children were studying in $10^{\text {th }}$ class. In case of urban locality, 36.29 per cent of children were studying in $8^{\text {th }}$ class followed by 34.68 per cent of children in $9^{\text {th }}$ class and 29.03 per cent of children in $10^{\text {th }}$ class. With respect to gender, in rural area, 56.74 per cent of victimized children were boys and 43.26 per cent of victimized children were girls. In case of urban locality, 58.06 per cent of victimized children were boys and 41.94 per cent of victimized children were girls.

The results regarding ordinal position, in rural locale, indicated that, majority $(53.19 \%)$ of victimized children were later born children followed by 46.81 per cent of children first born children. In urban locale, 53.23 per cent of victimized children were first born and 46.77 per cent of children were later born. The results showed that, with regard to academic achievement, in rural locality, 19.15 percent of children were $\mathrm{A}$ and $\mathrm{A}+$ graders, 47.52 per cent of children were $\mathrm{B}$ and $\mathrm{B}+$ graders and 33.33 per cent of victimized children were $C$ and $\mathrm{C}+$ graders. In case of urban locality, 25.00 percent of children were A and A+ graders, 49.19 per cent of victimized children were $\mathrm{B}$ and $\mathrm{B}+$ graders and 25.81 per cent of victimized children were $\mathrm{C}$ and $\mathrm{C}+$ graders.
The results of type of school revealed that, in rural locale, most of $(53.19 \%)$ the victimized children were studying in government schools followed by 46.81 per cent of victimized children were belonged to private schools. In case of urban locale, 54.03 per cent of children were studying in government schools and 45.97 per cent of victimized children were studying in private schools.

Distribution of phase 2 victimized rural and urban high school children based on parental characteristics are presented in table $3 \mathrm{~b}$. With regard to father's education, 53.19 per cent of fathers in rural locality had completed $10^{\text {th }}$ pass but $\leq$ graduation followed by primary pass but $\leq 10^{\text {th }}(27.66 \%)$, less than primary but attended school at least one year $(15.60 \%)$ and only 3.55 per cent of them had completed graduation. In case of urban area, most of them $(46.77 \%)$ had completed $10^{\text {th }}$ pass but $\leq$ graduation followed by less than primary but attended school at least one year $(25.81 \%)$, primary pass but $\leq 10^{\text {th }}(25.00 \%)$ and only 2.42 per cent of them completed graduation. None of the fathers were found in the category of post graduation and professional qualification with degree both in rural and urban areas.

Table.1 Percentage distribution of rural and urban high school children by personal characteristics

\begin{tabular}{|c|c|c|c|c|c|c|c|c|}
\hline \multirow{2}{*}{$\begin{array}{l}\text { Sl. } \\
\text { No }\end{array}$} & \multirow[t]{2}{*}{ Characteristics } & \multirow[t]{2}{*}{ Category } & \multicolumn{2}{|c|}{ Rural (582) } & \multicolumn{2}{|c|}{ Urban (568) } & \multicolumn{2}{|c|}{ Total (1150) } \\
\hline & & & $\mathbf{n}$ & $\%$ & $\mathbf{n}$ & $\%$ & $\mathbf{n}$ & $\%$ \\
\hline \multirow[t]{4}{*}{1} & Class & $8^{\text {th }}$ & 199 & 34.20 & 194 & 34.15 & 393 & 34.17 \\
\hline & & $9^{\text {th }}$ & 194 & 33.33 & 190 & 33.45 & 384 & 33.40 \\
\hline & & $10^{\text {th }}$ & 189 & 32.47 & 184 & 32.40 & 373 & 32.43 \\
\hline & & Total & 582 & 100 & 568 & 100 & 1150 & 100 \\
\hline \multirow[t]{3}{*}{2} & \multirow[t]{3}{*}{ Gender } & Boys & 296 & 50.86 & 285 & 50.18 & 581 & 50.52 \\
\hline & & Girls & 286 & 49.14 & 283 & 49.82 & 569 & 49.47 \\
\hline & & Total & 582 & 100 & 568 & 100 & 1150 & 100 \\
\hline \multirow[t]{3}{*}{3} & \multirow[t]{3}{*}{ Type of school } & Government & 278 & 47.77 & 288 & 50.70 & 566 & 49.22 \\
\hline & & Private & 304 & 52.23 & 280 & 49.30 & 584 & 50.78 \\
\hline & & Total & 582 & 100 & 568 & 100 & 1150 & 100 \\
\hline
\end{tabular}


Table. 2 Prevalence of peer victimization among rural and urban high school children

\begin{tabular}{|c|c|c|c|c|c|c|c|c|c|c|c|c|c|c|c|c|c|}
\hline \multirow{3}{*}{$\begin{array}{l}\text { Type of } \\
\text { school }\end{array}$} & \multirow[t]{3}{*}{ Class } & \multirow[t]{3}{*}{ Gender } & \multicolumn{5}{|c|}{ Rural (582) } & \multicolumn{5}{|c|}{ Urban (568) } & \multicolumn{5}{|c|}{ Total (1150) } \\
\hline & & & \multirow[t]{2}{*}{$\begin{array}{c}\text { Class } \\
\text { Strength }\end{array}$} & \multicolumn{2}{|c|}{$\begin{array}{c}\text { Peer } \\
\text { victimized } \\
\text { children }\end{array}$} & \multicolumn{2}{|c|}{ Total } & \multirow[t]{2}{*}{$\begin{array}{c}\text { Class } \\
\text { Strength }\end{array}$} & \multicolumn{2}{|c|}{$\begin{array}{c}\text { Peer } \\
\text { victimized } \\
\text { children }\end{array}$} & \multicolumn{2}{|c|}{ Total } & \multirow[t]{2}{*}{$\begin{array}{c}\text { Class } \\
\text { Strength }\end{array}$} & \multicolumn{2}{|c|}{$\begin{array}{c}\text { Peer } \\
\text { victimized } \\
\text { children }\end{array}$} & \multicolumn{2}{|c|}{ Total } \\
\hline & & & & $\mathbf{n}$ & $\%$ & $\mathbf{n}$ & $\%$ & & $\mathbf{n}$ & $\%$ & $\mathbf{n}$ & $\%$ & & $\mathbf{n}$ & $\%$ & $\mathbf{n}$ & $\%$ \\
\hline \multirow[t]{6}{*}{ Government } & $8^{\text {th }}$ & Boys & 50 & 15 & 5.66 & \multirow[t]{2}{*}{27} & \multirow[t]{2}{*}{$\begin{array}{c}10.1 \\
8\end{array}$} & 50 & 14 & 5.28 & \multirow[t]{2}{*}{24} & \multirow[t]{2}{*}{9.05} & 100 & 29 & $\begin{array}{c}10.9 \\
4\end{array}$ & \multirow[t]{2}{*}{51} & \multirow[t]{2}{*}{$\begin{array}{c}19.2 \\
4\end{array}$} \\
\hline & & Girls & 45 & 12 & 4.52 & & & 49 & 10 & 3.77 & & & 94 & 22 & 8.30 & & \\
\hline & & Girls & 45 & 11 & 4.16 & 26 & 9.82 & 48 & 10 & 3.77 & 24 & 9.05 & 93 & 21 & 7.92 & 50 & $\begin{array}{c}18.8 \\
6\end{array}$ \\
\hline & $10^{\text {th }}$ & Boys & 46 & 12 & 4.53 & \multirow[t]{2}{*}{22} & \multirow[t]{2}{*}{8.30} & 47 & 11 & 4.16 & \multirow[t]{2}{*}{19} & 7.18 & 93 & 23 & 8.68 & 41 & 15.4 \\
\hline & & Girls & 44 & 10 & 3.77 & & & 46 & 8 & 3.02 & & & 90 & 18 & 6.80 & & 8 \\
\hline & & Total & 278 & 75 & $\begin{array}{c}28.3 \\
0\end{array}$ & 75 & $\begin{array}{c}28.3 \\
0\end{array}$ & 288 & 67 & $\begin{array}{c}25.2 \\
8\end{array}$ & 67 & $\begin{array}{c}25.2 \\
8\end{array}$ & 566 & 142 & $\begin{array}{c}53.5 \\
8\end{array}$ & 142 & $\begin{array}{c}53.5 \\
8\end{array}$ \\
\hline Private & $8^{\text {th }}$ & Boys & 52 & 14 & 5.28 & 24 & 9.05 & 47 & 12 & 4.52 & 21 & 7.92 & 99 & 26 & 9.81 & 45 & 16.9 \\
\hline & & Girls & 52 & 10 & 3.77 & & & 48 & 9 & 3.40 & & & 100 & 19 & 7.17 & & 8 \\
\hline & & Girls & 51 & 10 & 3.77 & & & 47 & 8 & 3.02 & & & 98 & 18 & 6.80 & & 6 \\
\hline & $10^{\text {th }}$ & Boys & 50 & 11 & 4.16 & 19 & 7.18 & 46 & 10 & 3.77 & 17 & $6 . .41$ & 96 & 21 & 7.92 & 36 & 13.5 \\
\hline & & Girls & 49 & 08 & 3.02 & & & 45 & 7 & 2.64 & & & 94 & 15 & 5.66 & & 8 \\
\hline & & Total & 304 & 66 & $\begin{array}{c}24.9 \\
1\end{array}$ & 66 & $\begin{array}{c}24.9 \\
1\end{array}$ & 280 & 57 & $\begin{array}{c}21.5 \\
1\end{array}$ & 57 & $\begin{array}{c}21.5 \\
1\end{array}$ & 584 & 123 & $\begin{array}{c}46.4 \\
2\end{array}$ & 123 & $\begin{array}{c}46.4 \\
2\end{array}$ \\
\hline Total & & & 582 & 141 & $\begin{array}{c}53.2 \\
1\end{array}$ & $\begin{array}{c}14 \\
1\end{array}$ & $\begin{array}{c}53.2 \\
1\end{array}$ & 568 & 124 & $\begin{array}{c}46.7 \\
9\end{array}$ & $\begin{array}{c}12 \\
4\end{array}$ & $\begin{array}{c}46.7 \\
9\end{array}$ & 1150 & 265 & 100 & 265 & 100 \\
\hline
\end{tabular}


Table.3a Percentage distribution of victimized rural and urban high school children by personal characteristics

\begin{tabular}{|c|c|c|c|c|c|c|c|c|}
\hline \multirow{3}{*}{$\begin{array}{l}\text { Sl } \\
\text { No }\end{array}$} & \multirow{3}{*}{ Characteristics } & \multirow{3}{*}{ Category } & & & \multicolumn{4}{|c|}{$\mathrm{N}=265$} \\
\hline & & & \multicolumn{2}{|c|}{ Rural (141) } & \multicolumn{2}{|c|}{ Urban (124) } & \multicolumn{2}{|c|}{ Total } \\
\hline & & & $\mathbf{n}$ & $\%$ & $\mathbf{n}$ & $\%$ & $\mathbf{n}$ & $\%$ \\
\hline \multirow[t]{4}{*}{1} & \multirow[t]{4}{*}{ Class } & $8^{\text {th }}$ & 51 & 36.17 & 45 & 36.29 & 96 & 36.23 \\
\hline & & $9^{\text {th }}$ & 49 & 34.75 & 43 & 34.68 & 92 & 34.72 \\
\hline & & $10^{\text {th }}$ & 41 & 29.08 & 36 & 29.03 & 77 & 29.05 \\
\hline & & Total & 141 & 100 & 124 & 100 & 265 & 100 \\
\hline \multirow[t]{3}{*}{2} & \multirow[t]{3}{*}{ Gender } & Boys & 80 & 56.74 & 72 & 58.06 & 152 & 57.36 \\
\hline & & Girls & 61 & 43.26 & 52 & 41.94 & 113 & 42.64 \\
\hline & & Total & 141 & 100 & 124 & 100 & 265 & 100 \\
\hline \multirow[t]{3}{*}{3} & \multirow{3}{*}{$\begin{array}{l}\text { Ordinal } \\
\text { position }\end{array}$} & First born & 66 & 46.81 & 66 & 53.23 & 132 & 49.81 \\
\hline & & Later born & 75 & 53.19 & 58 & 46.77 & 133 & 50.19 \\
\hline & & Total & 141 & 100 & 124 & 100 & 265 & 100 \\
\hline \multirow[t]{4}{*}{4} & \multirow{4}{*}{$\begin{array}{c}\text { Academic } \\
\text { achievement }\end{array}$} & $\mathrm{A}$ and $\mathrm{A}+$ & 27 & 19.15 & 31 & 25.00 & 58 & 21.88 \\
\hline & & $\mathrm{B}$ and $\mathrm{B}+$ & 67 & 47.52 & 61 & 49.19 & 128 & 48.30 \\
\hline & & $\mathrm{C}$ and $\mathrm{C}+$ & 47 & 33.33 & 32 & 25.81 & 79 & 29.82 \\
\hline & & Total & 141 & 100 & 124 & 100 & 265 & 100 \\
\hline \multirow[t]{3}{*}{5} & \multirow[t]{3}{*}{ Type of school } & Government & 75 & 53.19 & 67 & 54.03 & 142 & 53.58 \\
\hline & & Private/Aided & 66 & 46.81 & 57 & 45.97 & 123 & 46.42 \\
\hline & & Total & 141 & 100 & 124 & 100 & 265 & 100 \\
\hline
\end{tabular}


Table.3b Percentage distribution of victimized rural and urban high school children by parental characteristics

\begin{tabular}{|c|c|c|c|c|c|c|c|c|}
\hline \multirow{3}{*}{$\begin{array}{l}\text { Sl. } \\
\text { No }\end{array}$} & \multirow{3}{*}{ Characteristics } & \multirow{3}{*}{ Category } & & & \multicolumn{4}{|c|}{$\mathrm{N}=265$} \\
\hline & & & \multicolumn{2}{|c|}{ Rural (141) } & \multicolumn{2}{|c|}{ Urban (124) } & \multicolumn{2}{|c|}{ Total } \\
\hline & & & $\mathbf{n}$ & $\%$ & $\mathbf{n}$ & $\%$ & $\mathbf{n}$ & $\%$ \\
\hline \multirow[t]{8}{*}{1} & \multirow[t]{8}{*}{ Father education } & Professional qualification with degree & - & & - & & & \\
\hline & & Post graduation & - & & - & & & \\
\hline & & Graduation & 5 & 3.55 & 3 & 2.42 & 8 & 3.02 \\
\hline & & $10^{\text {th }}$ pass but $\leq$ graduation & 75 & 53.19 & 58 & 46.77 & 133 & 50.18 \\
\hline & & Primary pass but $\leq 10^{\text {th }}$ & 39 & 27.66 & 31 & 25.00 & 70 & 26.42 \\
\hline & & $\begin{array}{c}\leq \text { Primary but attended school at least } \\
\text { one year }\end{array}$ & 22 & 15.60 & 32 & 25.81 & 54 & 20.38 \\
\hline & & Just literate but no schooling & - & & - & & - & \\
\hline & & Illiterate & - & & - & & - & \\
\hline \multirow[t]{8}{*}{2} & \multirow[t]{8}{*}{ Mother education } & $\begin{array}{l}\text { Professional qualification with degree } \\
\text { technical degree }\end{array}$ & - & & - & & - & \\
\hline & & Post graduation & - & & - & & - & \\
\hline & & Graduation & 3 & 2.13 & 4 & 3.22 & 7 & 2.64 \\
\hline & & $10^{\text {th }}$ pass but $\leq$ graduation & 26 & 18.45 & 36 & 29.03 & 62 & 23.39 \\
\hline & & Primary pass but $\leq 10^{\text {th }}$ & 56 & 39.71 & 36 & 29.03 & 92 & 34.72 \\
\hline & & $\begin{array}{l}\leq \text { Primary but attended school at least } \\
\text { one year }\end{array}$ & 56 & 39.71 & 48 & 38.72 & 104 & 39.25 \\
\hline & & Just literate but no schooling & - & & - & & - & \\
\hline & & Illiterate & - & & - & & - & \\
\hline \multirow[t]{6}{*}{3} & \multirow[t]{6}{*}{ Father occupation } & Service in central/state/public/undertaken & 7 & 04.96 & 2 & 01.61 & 9 & 03.40 \\
\hline & & Service in private sector /business & - & & 14 & 11.29 & 14 & 05.28 \\
\hline & & $\begin{array}{l}\text { Service at shops, transports, own } \\
\text { cultivation of land }\end{array}$ & 72 & 51.06 & 32 & 25.81 & 104 & 39.25 \\
\hline & & Self employment with income $\geq 5000$ & 30 & 21.28 & 41 & 33.06 & 71 & 26.79 \\
\hline & & $\begin{array}{l}\text { Self employment with income } \leq \\
5000 / \text { labourer }\end{array}$ & 32 & 22.70 & 35 & 28.23 & 67 & 25.28 \\
\hline & & Unemployment & - & & - & & - & \\
\hline \multirow[t]{6}{*}{4} & \multirow{6}{*}{$\begin{array}{l}\text { Mother } \\
\text { occupation }\end{array}$} & Service in central/state/public/undertaken & 2 & 01.42 & 2 & 01.61 & 4 & 01.51 \\
\hline & & Service in private sector /business & - & & - & & - & \\
\hline & & $\begin{array}{l}\text { Service at shops, transports, own } \\
\text { cultivation of land }\end{array}$ & 13 & 09.22 & 13 & 10.48 & 26 & 09.81 \\
\hline & & Self employment with income $\geq 5000$ & 16 & 11.35 & 29 & 23.39 & 45 & 16.98 \\
\hline & & $\begin{array}{l}\text { Self employment with income } \leq \\
5000 / \text { labourer/housewife }\end{array}$ & 110 & 78.01 & 80 & 64.52 & 190 & 71.70 \\
\hline & & Unemployment & - & & - & & - & \\
\hline
\end{tabular}


Table.3c Percentage distribution of victimized rural and urban high school children by familial characteristics

\begin{tabular}{|c|c|c|c|c|c|c|c|c|}
\hline \multirow{2}{*}{$\begin{array}{l}\text { Sl. } \\
\text { No }\end{array}$} & \multirow[t]{2}{*}{ Characteristics } & \multirow[t]{2}{*}{ Category } & \multicolumn{2}{|c|}{ Rural (141) } & \multicolumn{2}{|c|}{ Urban (124) } & \multicolumn{2}{|c|}{ Total } \\
\hline & & & $\mathbf{n}$ & $\%$ & n & $\%$ & $\mathbf{n}$ & $\%$ \\
\hline \multirow[t]{2}{*}{1} & \multirow{2}{*}{$\begin{array}{l}\text { Type of } \\
\text { family }\end{array}$} & Nuclear & 71 & 50.35 & 87 & 70.16 & 158 & 59.62 \\
\hline & & Joint & 70 & 49.65 & 37 & 29.84 & 107 & 40.38 \\
\hline \multirow[t]{3}{*}{2} & \multirow[t]{3}{*}{ Size of family } & $\begin{array}{l}\text { Small }(\leq 5 \\
\text { members })\end{array}$ & 72 & 51.06 & 87 & 70.16 & 159 & 60.00 \\
\hline & & $\begin{array}{l}\text { Medium (6-9 } \\
\text { members }\end{array}$ & 53 & 37.59 & 32 & 25.81 & 85 & 32.08 \\
\hline & & $\begin{array}{l}\text { Large }(\geq 9 \\
\text { members })\end{array}$ & 16 & 11.35 & 5 & 04.03 & 21 & 07.92 \\
\hline \multirow[t]{6}{*}{3} & \multirow{6}{*}{$\begin{array}{c}\text { Socio } \\
\text { economic } \\
\text { status }\end{array}$} & Upper high & - & & - & & - & \\
\hline & & High & 5 & 3.55 & 3 & 02.42 & 8 & 03.02 \\
\hline & & Upper middle & 11 & 07.80 & 14 & 11.29 & 25 & 09.43 \\
\hline & & Lower middle & 88 & 62.41 & 91 & 73.39 & 179 & 67.55 \\
\hline & & Poor & 37 & 26.24 & 16 & 12.90 & 53 & 20.00 \\
\hline & & Very poor & - & - & - & - & - & - \\
\hline \multirow[t]{4}{*}{4} & \multirow[t]{4}{*}{ Caste } & Upper caste & 23 & 16.31 & 27 & 21.77 & 50 & 18.87 \\
\hline & & $\begin{array}{c}\text { Other backward } \\
\text { caste }\end{array}$ & 42 & 29.79 & 43 & 34.67 & 85 & 32.07 \\
\hline & & Scheduled caste & 29 & 20.57 & 29 & 23.40 & 58 & 21.89 \\
\hline & & Scheduled tribe & 47 & 33.33 & 25 & 20.16 & 72 & 27.17 \\
\hline
\end{tabular}

Table.4a Levels of peer victimization among rural and urban high school children

\begin{tabular}{|c|c|c|c|c|c|c|c|}
\hline \multirow{3}{*}{$\begin{array}{l}\text { Levels of peer } \\
\text { victimization }\end{array}$} & \multicolumn{6}{|c|}{ Locality } & \multirow[t]{3}{*}{$\chi^{2}$} \\
\hline & \multicolumn{2}{|c|}{ Rural (141) } & \multicolumn{2}{|c|}{ Urban (124) } & \multicolumn{2}{|c|}{ Total (265) } & \\
\hline & $\mathbf{n}$ & $\%$ & $\mathbf{n}$ & $\%$ & n & $\%$ & \\
\hline Low & 27 & 19.15 & 37 & 29.84 & 64 & 24.15 & \multirow{4}{*}{$2.70^{\mathrm{NS}}$} \\
\hline Moderate & 96 & 68.08 & 73 & 58.87 & 169 & 63.77 & \\
\hline High & 18 & 12.77 & 14 & 11.29 & 32 & 12.08 & \\
\hline Total & 141 & 100 & 124 & 100 & 265 & 100 & \\
\hline
\end{tabular}

NS-Non Significant

Table.4b Comparison of peer victimization among rural and urban children

NS-Non Significant 
Table.5a Distribution of boys and girls by levels of peer victimization

\begin{tabular}{|c|c|c|c|c|c|c|c|}
\hline \multirow{3}{*}{$\begin{array}{l}\text { Levels of peer } \\
\text { victimization }\end{array}$} & \multirow{2}{*}{\multicolumn{2}{|c|}{ Boys (152) }} & \multirow{2}{*}{\multicolumn{2}{|c|}{ Girls (113) }} & \multirow{2}{*}{\multicolumn{2}{|c|}{ Total (265) }} & \multirow{3}{*}{$\begin{array}{c}\mathrm{N}=265 \\
\chi^{2}\end{array}$} \\
\hline & & & & & & & \\
\hline & $\mathbf{n}$ & $\%$ & $\mathbf{n}$ & $\%$ & $\mathbf{n}$ & $\%$ & \\
\hline Low & 35 & 23.03 & 29 & 25.66 & 64 & 24.15 & \multirow[t]{4}{*}{$10.90^{* *}$} \\
\hline Moderate & 90 & 59.21 & 79 & 69.91 & 169 & 63.77 & \\
\hline High & 27 & 17.76 & 5 & 4.43 & 32 & 12.08 & \\
\hline Total & 152 & 100 & 113 & 100 & 265 & 100 & \\
\hline
\end{tabular}

**Significant at 1 per cent level

Table.5b Comparison of peer victimization among boys and girls

*Significant at 5 per cent level

Table.6a Distribution of high school children by levels of peer victimization

\begin{tabular}{|c|c|c|c|c|c|c|c|c|c|}
\hline \multirow{3}{*}{$\begin{array}{l}\text { Levels of peer } \\
\text { victimization }\end{array}$} & \multirow{2}{*}{\multicolumn{2}{|c|}{$8^{\text {th }}(96)$}} & \multirow{2}{*}{\multicolumn{2}{|c|}{$9^{\text {th }}(92)$}} & \multirow{2}{*}{\multicolumn{2}{|c|}{$10^{\text {th }}(77)$}} & \multirow{2}{*}{\multicolumn{2}{|c|}{ Total (265) }} & $\mathrm{N}=265$ \\
\hline & & & & & & & & & $\chi^{2}$ \\
\hline & $\mathbf{n}$ & $\%$ & n & $\%$ & $\mathbf{n}$ & $\%$ & n & $\%$ & \\
\hline Low & 16 & 16.66 & 26 & 28.26 & 22 & 28.57 & 64 & 24.15 & \\
\hline Moderate & 65 & 67.71 & 59 & 64.13 & 45 & 58.44 & 169 & 63.77 & $6.64^{\mathrm{NS}}$ \\
\hline High & 15 & 15.63 & 7 & 7.61 & 10 & 12.99 & 32 & 12.08 & \\
\hline Total & 96 & 100 & 92 & 100 & 77 & 100 & 265 & 100 & \\
\hline
\end{tabular}

NS- Non Significant

Table.6b Comparison of peer victimization among high school children

\begin{tabular}{|c|c|c|c|c|}
\hline & $8^{\text {th }}(96)$ & $9^{\text {th }}(92)$ & $10^{\text {th }}(77)$ & $\begin{array}{c}\mathrm{N}=265 \\
\text { value }\end{array}$ \\
\hline Peer victimization & Mean \pm SD & Mean \pm SD & Mean \pm SD & $7.17^{* *}$ \\
\cline { 2 - 4 } & $23.36 \pm 3.80$ & $21.92 \pm 3.64$ & $21.20 \pm 4.12$ & \\
\hline
\end{tabular}

**Significant at 1 per cent level

The results related to mother's education showed that, in rural area, 39.71 per cent of them had completed $\leq$ primary but attended school at least one year and primary pass but $\leq$ $10^{\text {th }}$ equally followed by 18.45 per cent of them had completed $10^{\text {th }}$ pass but $\leq$ graduation and only 2.13 per cent of them had completed graduation. With regard to urban locality,
38.72 per cent of them had completed $\leq$ primary but attended school at least one year followed by primary pass but $\leq 10^{\text {th }}$ and $10^{\text {th }}$ pass but $\leq$ graduation $(29.03 \%)$ equally and only 3.22 percent of them had completed graduation. None of the mothers were found in the category of post graduation and professional qualification with degree in both 
rural and urban areas. The results related to father's occupation among rural area revealed that, 51.06 per cent of them had service at shops, transports, own cultivation of land followed by self employment with income $\leq$ 5000 (22.70\%), self employment with income $\geq 5000(21.28 \%)$ and only 4.96 per cent of them had service in central/state/public/undertaken. In case of urban locality most of them had self employment with income $\geq 5000$ (33.06\%), followed by self employment with income $\leq$ $5000 /$ labourer $(28.23 \%)$, services at shops, transports, own cultivation of land $(25.81 \%)$, service in private sector/business $(11.29 \%)$ and only 1.61 per cent of them had service in central/state/public/undertaken.

When mother's occupation was taken into account it was found that, in rural area majority $(78.01 \%)$ of the them had self employment with income s 5000/labourer/housewife followed by self employment with income $\geq 5000$ (11.35\%), service at shops, transports, own cultivation of land $(9.22 \%)$ and only 1.42 per cent of them had service at central/state/public/undertaken. In case of urban area 64.52 per cent of them had self employment with income $\leq$ 5000/labourer/ housewife followed by self employment with income $\geq 5000(23.39 \%)$, service at shops, transports, own cultivation of land $(10.48 \%)$ and only 1.61 per cent of them had service at central/ state/ public/ undertaken.

Results related to distribution of phase 2 victimized rural and urban high school children based on familial characteristics are presented in table $3 \mathrm{c}$. The results in relation to type of family shows that, in rural area, 50.35 per cent of children were belonged to nuclear family and 49.65 per cent of children were belonged joint family. In case of urban area, majority of children were came from nuclear family $(70.16 \%)$ followed by 29.84 per cent of children were belonged to joint family.
With respect to size of family it was found that, most of children were from small family $(51.06 \%)$ followed by 37.59 per cent of children were belonged to medium family and only 11.35 per cent of children were belonged to large family. In case of urban areas, 70.16 per cent of children were belonged to small family, 25.81 per cent of children were from medium family and only 4.03 per cent of children were belongs large family.

The results with regard to socio economic status revealed that, in rural area, 62.41 per cent of children were from lower middle class family, 26.24 per cent of children were from poor class family, 7.80 per cent of children were from upper middle class and only 3.55 per cent of children were from higher socio economic status family. In case of urban area majority of children were from lower middle class family $(73.39 \%)$ followed by poor class family (12.90\%), upper middle class family $(11.29 \%)$ and only 2.42 per cent of children were from higher socio economic status family. When children's caste was taken into account it was found that, in rural 33.33 per cent of children belonged to scheduled tribe, 20.57 per cent of children were scheduled caste, 29.79 per cent of children were other backward caste and only 16.31 per cent of children were upper caste. In case of urban area, most $(34.67 \%)$ of children were other backward caste followed by scheduled caste $(23.40 \%)$, upper caste $(21.77 \%)$ and scheduled tribe $(20.16 \%)$.

The levels of peer victimization among rural and urban high school children presented in table 4a shows that, in rural area, majority of children $(68.08 \%)$ were found in moderate level of victimization and 19.15 per cent of children were found in low level of victimization. About 12.77 per cent children were found to be highly victimized. In case of urban area, majority of children were found to be moderately victimized $(58.87 \%)$ followed by low level of victimization $(29.84 \%)$. 
However statistical analysis indicates a non significant association between locality and levels of victimization was observed.

Results related to comparison between peer victimization among rural and urban children presented in table $4 \mathrm{~b}$. reveal that, children from both rural and urban area were found to be equally victimized. Hence, no significant difference was found between rural and urban children. Results of the study Guo et al., (2010) also revealed that, there was no statistical significance in peer victimization between urban $(8.80 \%)$ and rural $(8.40 \%)$ Chinese school children.

The results of the table 5a shows that, most of boys $(59.21 \%)$ and girls $(69.91 \%)$ were found in moderate level of victimization followed by low level of victimization was experienced by 23.03 per cent of boys and 25.66 per cent of girls. About 17.76 per cent of boys and 4.43 per cent of girls were highly victimized. On analysis a significant association was found between gender and levels of victimization among children.

Results related to comparison of peer victimization among boys and girls presented in table $5 \mathrm{~b}$. reveal that, boys were found to be significantly more (22.60) victimized compared to girls (21.74). The findings of the present study are in line with Adeosun et al., (2015) reported that, males significantly more $(63.4 \%)$ victimized than females $(47.4 \%)$.

The distribution of high school children by levels of peer victimization presented in table 6a shows that, most of children studying $8^{\text {th }}$ class $(65.71 \%)$ were fell under moderate level of victimization while, 28.26 per cent of $9^{\text {th }}$ class children and 28.57 per cent of $10^{\text {th }}$ class children were found in low level of victimization. On analysis a significant association was found between class and levels of peer victimization among children.
Results related to comparison between peer victimization among high school children presented in table $6 \mathrm{~b}$ reveal that, children studying in $8^{\text {th }}$ class were significantly more (23.36) victimized compared to children studying in $9^{\text {th }}$ class (21.92). Abdulsalam et al., (2017) reported similar results that, children studying in $7^{\text {th }}$ grade were 1.65 times significantly higher risk for victim compared to $8^{\text {th }}$ grade.

Prevalence of peer victimization was found more among rural children. In rural area, children studying in government schools were more victimized than the private schools children. In government schools, boys studying in $8^{\text {th }}$ class were found to be more victimized than other classes. Rural children had experienced high and moderate level of victimization while, urban children had low level of victimization. Association and difference between peer victimization among rural and urban children was found non significant. So, there is need for school based educational programs and public awareness campaigns, including print, electronic and social media with emphasis to rural and urban area and strict enforcement of policies to make the school environment safe is necessary.

\section{Acknowledgement}

This research was supported by College of Community Science UAS Dharwad. I would like to express my gratitude to my major advisor for sharing their pearls of wisdom with me during the course of this research. I thank school authorities and children who participated in this study and my sisters and friends who helped in data collection.

\section{References}

Abdulsalam, A. J., Daihani, A. E. and Francis, K., 2017, Prevalence and associated factors of peer victimization (bullying) 
among grades 7 and 8 middle school students in Kuwait. International Journal of Pediatrics, 1 (1): 1-8.

Adeosun, I. I., Abosede, A., Jejeloye, A., Oyekunle, O., Ogunlowo, O. and Pedro, A., 2015, Bullying victimization among secondary school students in Lagos, Nigeria: Emotional, behavioral and mental health correlates. British Journal of Education Society and Behavioural Science, 11 (1): 1-8.

Anonymous., 2019, Bully back off: statistics. 6-9.

Anonymous., 2018, An everyday lesson: End violence in schools, UNICEF, New York.

Dake, J. A., Price, J. H. and Telljohann, S. K., 2003, The nature and extent of bullying at school. Journal of School Health, 73(5):173-180.

Delfabbro, P., Winefield, T., Trainor, S., Dollard, M., Anderson, S., Metzer, J. and Hammarstrom, A., 2006, Peer and teacher bullying/victimization of South Australian secondary school students: prevalence and psychosocial profiles.
British Journal of Educational Psychology, 76:71-90.

Finkelhore, D., Ormord, R. K. and Turner, H. A., 2007, Poly victimization: A neglected component in child victimization. Child Abuse and Neglect. 31:7-26.

Guo, Q. Z., Ma, W. J., Nie, S. P., Xu, Y. J., $\mathrm{Xu}, \mathrm{H}$. F. and Zhang, Y. R., 2010, Relationships between weight status and bullying victimization among school age adolescents in Guangdong province of China. Biomedical and Environmental Science, 23:108-112.

Mynard, H. and Joseph, S., 2000, Development of the multidimensional peer victimization scale. Aggressive Behavior, 26:169-178.

Olweus, D., 1993, Bullying at school: what we know and what we can do. Oxford, UK: Blackwell.

Ramya, S. G., Kulkarni, M. L., 2011, Bullying among school children: prevalence and association with common symptoms in childhood. Indian Journal of Pediatrics. 78(3):307-310.

\section{How to cite this article:}

Drakshayini T. Tippanagoudra, Manjula Patil and Saraswati Hunshal. 2020. Peer Victimization among High School Children: A Prevalence Study. Int.J.Curr.Microbiol.App.Sci. 9(03): 30893101. doi: https://doi.org/10.20546/ijcmas.2020.903.354 